\title{
Microchimerism of maternal origin persists into adult life
}

\author{
Sean Maloney, ${ }^{1}$ Anajane Smith, ${ }^{1}$ Daniel E. Furst, ${ }^{2}$ David Myerson, ${ }^{4}$ Kate Rupert,${ }^{3}$ \\ Paul C. Evans, ${ }^{5}$ and J. Lee Nelson ${ }^{1,6}$ \\ ${ }^{1}$ Immunogenetics Program, Fred Hutchinson Cancer Research Center, Seattle, Washington 98109, USA \\ ${ }^{2}$ Arthritis Clinical Research Unit, Virginia Mason Research Center, Seattle, Washington 98101, USA \\ ${ }^{3}$ Molecular Cytogenetics, Fred Hutchinson Cancer Research Center, Seattle, Washington 98109, USA \\ ${ }^{4}$ Department of Pathology, University of Washington, Seattle, Washington 98195, USA \\ ${ }^{5}$ Department of Immunology, The Babraham Institute, Cambridge CB2 4AT, United Kingdom \\ ${ }^{6}$ Division of Rheumatology, University of Washington, Seattle, Washington 98195, USA
}

Address correspondence to: J. Lee Nelson, Immunogenetics Program, Fred Hutchinson Cancer Research Center, 1100 Fairview, Avenue North, Seattle, Washington 98109, USA. Phone: (206) 667-5149; Fax: (206) 667-5255.

Received for publication February 19, 1999, and accepted in revised form May 17, 1999.

Recent studies indicate that fetal cells persist in maternal blood for decades after pregnancy. Maternal cells are known to engraft and persist in infants with immunodeficiency, but whether maternal cells persist long-term in immunocompetent offspring has not specifically been investigated. We developed sensitive human leukocyte antigen-specific (HLA-specific) PCR assays and targeted nonshared maternal HLA genes to test for persistent maternal microchimerism in subjects with scleroderma and in healthy normal subjects. Nonshared maternal-specific DNA was found in 6 of 9 scleroderma patients. In situ hybridization with double labeling for $\mathrm{X}$ and $\mathrm{Y}$ chromosome-specific sequences revealed female cells in peripheral blood samples from 2 male scleroderma patients. HLAspecific PCR also frequently revealed persistent maternal microchimerism in healthy control subjects. The mean age of all subjects with maternal microchimerism was 28 years (range: 9-49 years). With few exceptions, mothers of subjects with persistent maternal microchimerism were HLA incompatible with subjects for class I and class II alleles. These results clearly indicate that HLA-disparate maternal cells can persist in immunocompetent offspring well into adult life. The biological significance of maternal microchimerism and whether it might contribute to autoimmune disease requires further investigation.

J. Clin. Invest. 104:41-47 (1999).

\section{Introduction}

The placenta was traditionally thought to be a barricade that keeps the genetically differing mother and child separate. This assumption has recently given way, as a result of the application of molecular biological techniques to the study of human pregnancy. It is now recognized that fetal cells routinely pass into the maternal circulation during normal human pregnancy (1). Maternal cells can also be found in the fetal circulation (1, 2). In 1996, Bianchi et al. (3) reported the surprising observation that fetal cells persist in the maternal circulation for decades after pregnancy completion. Maternal cells are known to engraft and persist in infants with immunodeficiency (4). However, whether maternal cells persist long term in immunocompetent offspring, or are a factor in subsequent autoimmune disease, has not been investigated previously.

We studied patients with scleroderma because the hypothesis has been proposed that microchimerism is involved in autoimmune diseases such as scleroderma (5). The hypothesis was based on clinical similarities of scleroderma and chronic graft-versus-host disease (known to involve chimerism), the increased incidence of scleroderma in middle-aged women, and the longterm persistence of fetal cells after pregnancy comple- tion. In previous studies, we found that persistent fetal microchimerism is more frequent and quantitatively greater in the peripheral blood of women with scleroderma than in normal women (6). However, scleroderma also occurs in men and in women who have never been pregnant. In the current studies, therefore, we sought to investigate whether maternal cells sometimes persist into adult life in immunocompetent offspring. Engraftments from blood transfusion (7) or from a twin (8) represent additional potential sources of microchimerism. We designed human leukocyte antigen-specific (HLA-specific) primers that target nonshared maternal-specific HLA alleles, and tested DNA extracted from the peripheral blood of patients with scleroderma and normal subjects.

\section{Methods}

Study subjects, clinical specimens, and HLA genotyping. All subjects with a history of blood transfusion were excluded from the study. Thirty-two families with a history of simplex scleroderma were recruited and studied for HLA class II and class I alleles; 3 generations were studied for 20 families and 2 generations for 12 families. Three generations were required for study entry for all women with children, and participation of all chil- 


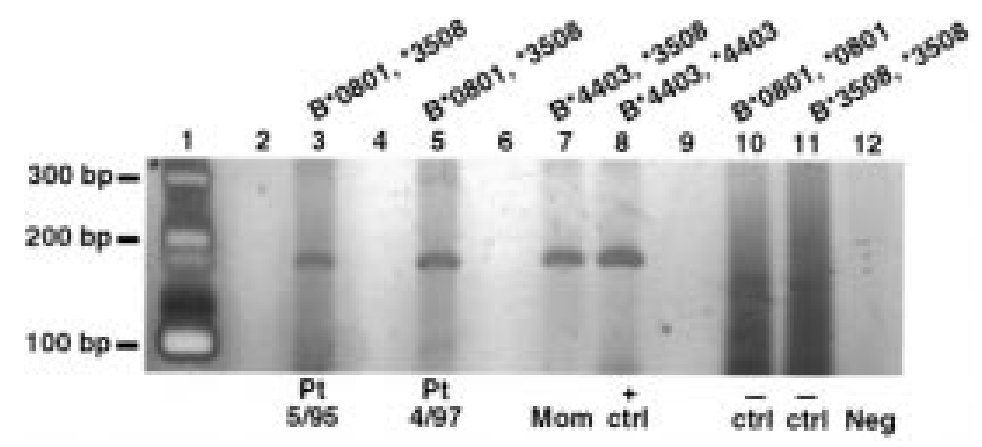

Figure 1

HLA-B44-specific PCR of DNA extracted from PBMCs of a 49-year-old scleroderma patient who was B44-negative and whose mother was B44-positive. Lanes 3 and 5 are patient samples from 2 different draw dates. Lane 7 is a positive control from the mother, and lane 8 is a positive control from a homozygous cell line. The patient's HLA-B alleles were $B^{*} 0801$ and $B * 3508$, and lanes 10 and 11 are negative controls derived from homozygous cell lines with these alleles. Lane 12 is a negative reagent control, and lanes 2, 4, 6, and 9 are blank.

Table 1

Sequencing result for the HLA-specific product shown above and deduced amino acid sequence

5'- CGC GGG TAT GAC CAG GAC GCC TAC GAC GGC AAG GAT TAC ATC GCC CTG AAC GAG GAC CTG AGC TCC TGG

$\begin{array}{llllllllllllllllllllllll}\text { R } & G & \text { Y } & \text { D } & \text { Q } & \text { D } & \text { A } & \text { Y } & \text { D } & \text { G } & \text { K } & \text { D } & \text { Y } & \text { I } & \text { A } & \text { L } & \text { N } & \text { E } & \text { D } & \text { L } & \text { S } & \text { S } & \text { W }\end{array}$

ACC GCG GCG GAC ACC GCG GCT CAG ATC ACC CAG CGC AAG TGG GAG GCG GCC CGT GTG GCG GAG CAG CTG

$\begin{array}{llllllllllllllllllllllllllllllllll}\mathrm{T} & \mathrm{A} & \mathrm{A} & \mathrm{D} & \mathrm{T} & \mathrm{A} & \mathrm{A} & \mathrm{Q} & \mathrm{I} & \mathrm{T} & \mathrm{Q} & \mathrm{R} & \mathrm{K} & \mathrm{W} & \mathrm{E} & \mathrm{A} & \mathrm{A} & \mathrm{R} & \mathrm{V} & \mathrm{A} & \mathrm{E} & \mathrm{Q} & \mathrm{L}\end{array}$

AGA GCC TAC CTG GAG GGC CTG TGC GTG GAG TCG CTC CGC AGA TAC CTG-3’

$\begin{array}{llllllllllllllll}\mathrm{R} & \mathrm{A} & \mathrm{Y} & \mathrm{L} & \mathrm{E} & \mathrm{G} & \mathrm{L} & \mathrm{C} & \mathrm{V} & \mathrm{E} & \mathrm{S} & \mathrm{L} & \mathrm{R} & \mathrm{R} & \mathrm{Y} & \mathrm{L}\end{array}$

The identical sequence was obtained from the patient and the mother, and corresponds to the specific allele $B * 4403$.

dren was required because microchimerism could derive from the mother or from a child in these subjects. Participation of men, children, and never-pregnant women included 2 generations (i.e., the subject and mother). One scleroderma patient was a twin; the unaffected twin was also recruited to the study. Thirtythree healthy control families were recruited, including 22 with 3 generations and 11 with 2 generations. HLA genotyping was completed for a total of 254 individuals: 128 in scleroderma families and 126 in controls. Some additional family members were included to confirm HLA haplotype assignments. From this database, subjects were selected for further study when the subject's mother had a nonshared HLA allele that was targeted by HLA-specific assays. All subjects granted informed consent as approved by the Fred Hutchinson Cancer Research Center Institutional Review Board.

PBMCs were isolated from whole heparinized blood by dilution in HBSS, followed by Ficoll-Hypaque (Pharmacia Biotech AB, Uppsala, Sweden) gradient centrifugation at $1.077 \mathrm{~g} / \mathrm{mL}$. For some children, DNA was extracted from hair roots for HLA typing as described previously (9). Genomic DNA was extracted from PBMCs using an Isoquick Nucleic Extraction Kit (Orca Research Inc., Bothell, Washington, USA), according to the manufacturer's instructions, and resuspended in 10 $\mathrm{mM}$ Tris- $\mathrm{HCl}$ ( $\mathrm{pH}$ 9.0). DNA quantity and purity were determined by standard spectrophotometric methods. For some subjects, DNA was extracted directly from whole blood samples.

DNA-based typing with sequence-specific oligonucleotide probe panels was used to identify specific alleles at the DRB1, DRB3, DRB4, DRB5, DQA1, and DQB1 loci. For $D R B 1$, an initial low-resolution assay detects $D R B 1$ families $D B R 1 * 01$ to $D R B 1 * 14$ and is followed by 1 or more high-resolution assays that identify specific $D R B 1$ alleles as described previously $(10,11) . D Q A 1$ and $D Q B 1$ alleles were determined using methods similar to those described previously (9), with additional probes added to detect newly identified alleles (12). HLA class I antigens were determined using a standard lymphocytotoxicity assay that discriminates 20 HLA-A, 30 HLA-B, and 8 HLA-Cw antigens. Some samples were subjected to sequencing to determine specific HLA class I alleles (13). To confirm HLA allele and antigen assignments and homozygosity, multiple family members were genotyped - including fathers and siblings of subjects, when available.

HLA-specific PCR primers. Primer sequences were designed based on all known allele sequences (12). Primer synthesis was done by Oligos Etc. (Wilsonville, Oregon, USA). One primer set was designed to target an HLA class I polymorphism and 3 targeted HLA class II polymorphisms. To target HLA-B44, a primer was designed that amplifies a group of HLA-B alleles based on polymorphisms at positions 66, 69, and 75 of exon 3 and another group of HLA-B alleles based on a polymorphism at position 229 of exon 3. The combination of primers specifically amplifies HLA-B44 alleles, producing a 190-bp fragment. All $D R B$-specific primers were designed to amplify allele groups based on sequence polymorphisms in the hypervariable region of exon 2. For $H L A-D R B 5^{*} 01$, one primer amplifies a group of $D R B 5^{*} 01$ alleles based on polymorphisms at positions $25,26,31,32$, and 38 , and the other amplifies a group of $D R B 5^{*} 01$ alleles based on polymorphisms between posi- 
tions 215 and 231 . The combination of primers specifically amplifies $H L A-D R B 5^{*} 01$ alleles, producing a 210 -bp fragment. For $H L A-D R B 1 * 04$, one primer amplifies a group of $D R B 1 * 04$ alleles based on polymorphisms at positions $25,26,31$, and 36 , and the other amplifies a group of $D R B 1 * 04$ alleles based on polymorphisms at positions 97 and 110 . The combination of primers specifically amplifies $D R B 1 * 04$, producing a 104-bp fragment. Further discrimination among $D R B 1 * 04$ alleles was achieved by using the former primer along with 1 of 2 primers that discriminate a dimorphism at position 258 (codon 86), producing a 263-bp fragment. For HLA$D R B 1 * 11$, one primer amplifies a group of $D R B 1 * 11$ alleles based on polymorphisms at positions $25,26,28,30$, 31,32 , and 35 of exon 2 , and the second primer amplifies a group of $D R B 1 * 11$ alleles based on polymorphisms at positions 173 and 174 of exon 2 . The combination of primers specifically amplifies $D R B 1 * 11$ and produces a 178-bp fragment. Primer sequences are as follows: B44specific primers 5'-TCC GCG GGT ATG ACC AGG A-3' and $3^{\prime}$-TCC AGG TAT CTG CGG AGC G-5'; DRB5*01specific primers $5^{\prime}$-TTC TTG CAG CAG GAT AAG TAT$3^{\prime}$ and $3^{\prime}$-GTA GTT GTC CAC CGC GGC G-5'; $D R B 1 * 04$-specific primers 5 '-CCA CGT TTC TTG GAG CAG GTT AAA-3' and 3'-GCG CAC GTA CTC CTC TTG GTG-5'; DRB $1 * 11$-specific primers $5{ }^{\prime}$-CA CGT TTC TTG GAG TAC TCT AC-3' and $5^{\prime}$-CTG GCT GTT CCA GTA CTC CT-5'.

HLA-specific PCR for maternal microchimerism. PCR reactions were performed in a volume of $50 \mu \mathrm{L}$ containing $1.25-1.5 \mu \mathrm{g}$ genomic DNA, $10 \mathrm{mmol} / \mathrm{L}$ Tris- $\mathrm{HCl}(\mathrm{pH}$ 8.3), $50 \mathrm{mmol} / \mathrm{L} \mathrm{KCl}, 1.5 \mathrm{mmol} \mathrm{MgCl}_{2}, 0.001 \% \mathrm{wt} / \mathrm{vol}$ gelatin, $260 \mu \mathrm{mol} / \mathrm{L}$ of each deoxynucleotide, $0.5 \mathrm{U}$ Perfect Match PCR Enhancer (Stratagene, La Jolla, California, USA), 2.5 U AmpliTaq Gold DNA polymerase (Perkin-Elmer Applied Biosystems, Foster City, California, USA), and 20 pmol of each HLA-specific primer. Ultrapure water was added to make up the final volume of $50 \mu \mathrm{L}$. Amplification consisted of 5 minutes at $96^{\circ} \mathrm{C}, 35$ cycles at $95^{\circ} \mathrm{C}$ for 35 seconds, and at $65^{\circ} \mathrm{C}$ for 35 seconds for HLA-B $44\left(58.5^{\circ} \mathrm{C}\right.$ for $D R B 5^{*} 01 ; 72^{\circ} \mathrm{C}$ for $D R B 1^{*} 04 ; 64.5^{\circ} \mathrm{C}$ for $D R B 1^{*} 11$ ), then $72^{\circ} \mathrm{C}$ for 1 minute, with a final extension step at $72^{\circ} \mathrm{C}$ for $10 \mathrm{~min}$ utes. Optimum amplification, sensitivity, and specificity were achieved by titrating $\mathrm{MgCl}_{2}$, and primer concentrations, number of thermocycles, and annealing temperature. All amplifications were performed in a GeneAmp System 9600 thermocycler (Perkin-Elmer Applied Biosystems). Assay sensitivity was determined by spiking experiments in which target DNA was serially diluted and added to a fixed amount of background DNA corresponding to alleles of the subject. The HLA-B44- and DRB ${ }^{*} 01$-specific PCR assays were able to detect at least 1 target cell in a background 500,000 cells; the HLA-DRB $1 * 04$ - and DRB $1 * 11$-specific PCR assays were able to detect at least 1 target cell in a background of 100,000 cells. Each PCR assay included the following controls: (a) a positive control of DNA from a cell line with the HLA allele of interest (0.2-0.5 $\mu \mathrm{g})$; (b) a positive control from the subject's mother $(0.2-0.5 \mu \mathrm{g}) ;(\mathrm{c})$ negative controls of DNA from a cell line with the same HLA alleles as the subject $(1.25 \mu \mathrm{g}$ and $0.35 \mu \mathrm{g})$; and (d) a negative control consisting of all PCR reagents without DNA. PCR products were electrophoresed at $100 \mathrm{~V}$ constant voltage for 1 hour in $6 \%$ or $8 \%$ precast TBE gels using a Novex Xcell II Mini-Cell (Novex, San Diego, California, USA). Each gel included positive and negative PCR controls and a 100-bp ladder (SLL-100; Gensura, Del Mar, California, USA). Gels were stained with silver stain (Silver Stain Plus Kit, BioRad Laboratories Inc., Hercules, California, USA) and dried with the DryEase Gel Drying System (Novex). DNA extracted from whole blood was tested in 4 samples of insufficient quantity to isolate PBMCs. All tests were done in duplicate, and most samples were assayed more than once.

$P C R$ precautionary measures. Extreme caution was employed to avoid and detect possible PCR contamination. Separate areas were used for PBMC separation, extraction of genomic DNA, PCR setup and amplification, and analysis of PCR products, with before-andafter PCR tests conducted in separate laboratories. Aerosol-resistant pipette tips (Molecular Bio-Products Inc., San Diego, California, USA) were used in all experiments, and multiple negative controls were included in all PCR amplifications.

Sequencing of HLA-specific PCR products. Seven microliters of the initial HLA-specific PCR product was subjected to an additional 40 cycles of amplification using the original thermocycling parameters. Thirty microliters of this PCR product was electrophoresed in a $1.75 \%$ ultrapure agarose gel (GIBCO BRL, Grand Island, New York, USA) and stained with a solution of ethidium bromide $(0.5 \mu \mathrm{g} / \mathrm{mL})$. The band was excised, eluted, and purified using a QIAquick Gel Extraction Kit (QIAGEN Inc., Valencia, California, USA). The purified PCR product was eluted into $30 \mu \mathrm{L}$ of $10 \mathrm{mmol}$ Tris- $\mathrm{HCl}$ ( $\mathrm{pH}$ 9.0), and $100 \mathrm{ng}$ was added to a reaction mix containing $8.0 \mu \mathrm{L}$ of sequencing reagent pre-mix (Thermo Sequenase; Amersham Corp., Arlington Heights, Illinois, USA), 12.5 pmol $5^{\prime}$ or $3^{\prime}$ primer, and $1 \mu \mathrm{L}$ DMSO (Sigma Chemical Co., St. Louis, Missouri, USA). Ultrapure water was added to make a final volume of $20 \mu \mathrm{L}$. The sequencing reaction mixtures were heated at $96^{\circ} \mathrm{C}$ for 3 minutes in a GeneAmp System 9600 thermocycler, followed by 25 cycles at $96^{\circ} \mathrm{C}$ for 10 seconds, $50^{\circ} \mathrm{C}$ for 5 seconds, and $60^{\circ} \mathrm{C}$ for 4 minutes. Resulting PCR products were column purified and electrophoresed as above. Sense and antisense sequences were determined in duplicate for the subject's and mother's HLA-specific PCR products, as well as for positive control cell line PCR products. Sequence analysis was performed using Wisconsin Package version 9.1 software from Genetics Computer Group (Madison, Wisconsin, USA).

Cytospin slide preparation and in situ bybridization. Cytospin slide preparations were analyzed for the presence of $\mathrm{X}$ and $\mathrm{Y}$ chromosome-specific sequences, using 


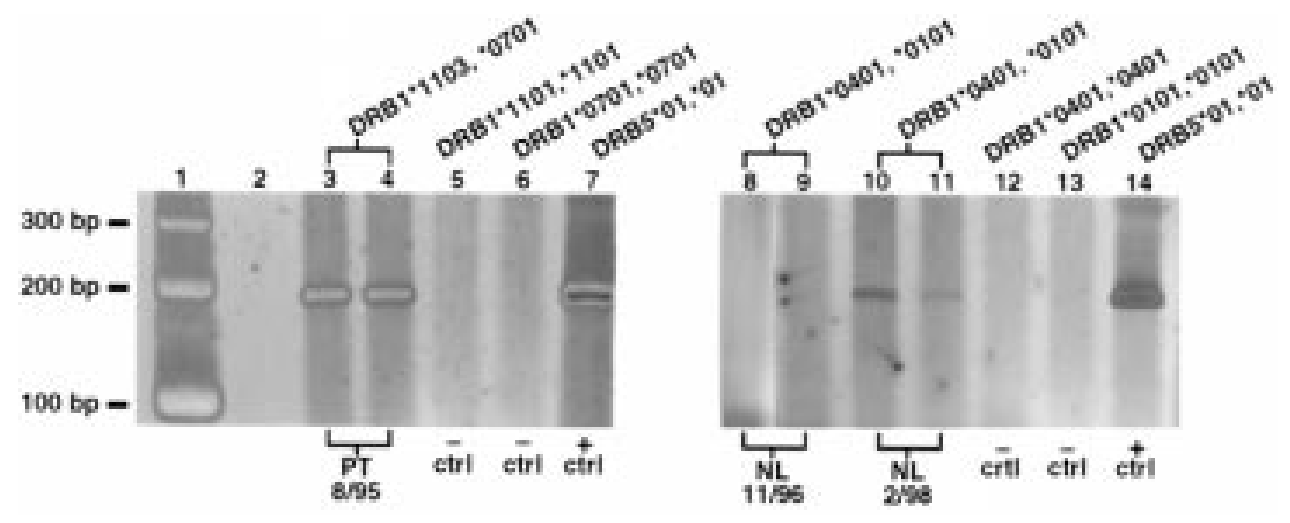

Figure 2

HLA-DRB5*01-specific PCR of DNA extracted from PBMCs of a scleroderma patient and a normal subject. The DRB5 gene is present only on some HLA haplotypes. Both mothers had $D R B 5^{*} 01$ and both subjects did not. Lanes 3 and 4 are patient samples (different aliquots from the same draw date). Negative controls from homozygous cell lines with alleles similar to the patient's are shown in lanes 5 and 6 , and a positive homozygous control is shown in lane 7. Lanes 8-11 are 2 draw dates from a normal subject. Lanes 12 and 13 are negative cell line controls, and lane 14 is a positive homozygous cell line control. Lane 2 is blank. There was no alternative source of DRB5*01 DNA in the normal subject. The scleroderma patient also had children with $D R B 5^{*} 01$ (see text).

Table 2

Sequencing result for the products of HLA-specific PCR shown above and deduced amino acid sequence

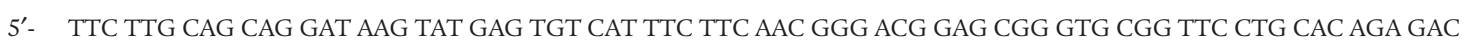

$\begin{array}{lllllllllllllllllllllllllll}\mathrm{F} & \mathrm{L} & \mathrm{Q} & \mathrm{Q} & \mathrm{D} & \mathrm{K} & \mathrm{Y} & \mathrm{E} & \mathrm{C} & \mathrm{H} & \mathrm{F} & \mathrm{F} & \mathrm{N} & \mathrm{G} & \mathrm{T} & \mathrm{E} & \mathrm{R} & \mathrm{V} & \mathrm{R} & \mathrm{F} & \mathrm{L} & \mathrm{H} & \mathrm{R} & \mathrm{D}\end{array}$

ATC TAT AAC CAA GAG GAG GAC TTG CGC TTC GAC AGC GAC GTG GGG GAG TAC CGG GCG GTG ACG GAG CTG GGG

$\begin{array}{llllllllllllllllllllllllll}\text { I } & \text { Y } & \text { N } & \text { Q } & \text { E } & \text { E } & \text { D } & \text { L } & \text { R } & \text { F } & \text { D } & \text { S } & \text { D } & \text { V } & \text { G } & \text { E } & \text { Y } & \text { R } & \text { A } & \text { V } & \text { T } & \text { E } & \text { L } & G\end{array}$

CGG CCT GAC GCT GAG TAC TGG AAC AGC CAG AAG GAC TTC CTG GAA GAC AGG CGC GCC GCG GTG GAC-3’ $\begin{array}{lllllllllllllllllllllllll}\mathrm{R} & \mathrm{P} & \mathrm{D} & \mathrm{A} & \mathrm{E} & \mathrm{Y} & \mathrm{W} & \mathrm{N} & \mathrm{S} & \mathrm{Q} & \mathrm{K} & \mathrm{D} & \mathrm{F} & \mathrm{L} & \mathrm{E} & \mathrm{D} & \mathrm{R} & \mathrm{R} & \mathrm{A} & \mathrm{A} & \mathrm{V} & \mathrm{D}\end{array}$

The identical sequence was obtained from the patient, the normal subject, and both mothers. The sequence corresponds to DRB5*0101.

a technique developed in the Fred Hutchinson Cancer Research Center Molecular Cytogenetics Laboratory for quantitative analysis of chimerism in sex-mismatched stem cell transplant pairs (14). Cytospin samples of male cells were prepared by centrifugation of 30,000-60,000 freshly separated PBMCs onto glass slides, $300 \mu \mathrm{L}$ per slide. Slides were stored in a desiccation chamber until use. The X chromosome-specific probe DXZ1 (15) was nick-translated with digoxigenin, and the $\mathrm{Y}$ chromosome-specific probe DYZ1 (16) was nick-translated with biotin. The XY probe mixture had a final concentration of $0.5 \mu \mathrm{g} / \mathrm{mL}$ of each probe in hybridization buffer of $50 \%$ formamide, $2 \times$ SSC $(0.3 \mathrm{M}$ sodium chloride and $0.03 \mathrm{M}$ sodium citrate [ $\mathrm{pH} 7.2]$ ), and $10 \%$ dextran sulfate. It was denatured at $72^{\circ} \mathrm{C}$ for 5 minutes, cooled on ice, and applied to the slide. Slides were digested in pepsin $(0.1 \mathrm{mg} / \mathrm{mL}$ in $0.01 \mathrm{M} \mathrm{HCl})$ at $37^{\circ} \mathrm{C}$ for 3 minutes, fixed in $4 \%$ buffered formalin for 15 minutes, rinsed in PBS, dehydrated in ethanol (70\%, 95\%, and 100\%), and air dried. Slides were denatured by treatment in $2 \times$ SSC $\left(72^{\circ} \mathrm{C}\right)$ for 10 minutes, $70 \%$ formamide, $2 \times \operatorname{SSC}\left(72^{\circ} \mathrm{C}\right)$ for 3 minutes, dehydrated in an ethanol series $\left(-20^{\circ} \mathrm{C}\right)$, and air dried. Ten microliters of probe was applied to the slide and then mounted under a coverslip $(22 \times 22 \mathrm{~mm})$ sealed with rubber cement. It was incubated overnight at $42^{\circ} \mathrm{C}$ in a humidified chamber. Slides were washed in $55 \%$ formamide, $2 \times \mathrm{SSC}\left(45^{\circ} \mathrm{C}\right)$ for 15 minutes, and $2 \times$ SSC (room temperature) for 5 minutes. Probe signals were simultaneously detected with fluorescein-coupled anti-digoxigenin (1:500 dilution; Boehringer Mannheim Biochemicals, Indianapolis, Indiana, USA) and Texas red-coupled avidin (1:500 dilution; Vector Laboratories, Burlingame, California, USA) for 30 minutes at room temperature in the dark. After washing with $2 \times$ SSC and PBS, they were mounted with VECTASHIELD (Vector Laboratories). Slides were evaluated directly using a Nikon E600 epifluorescence microscope with a 100-W mercury lamp equipped with appropriate single, double, and triple band-pass filters. Only cells with 2 visible chromosome signals were enumerated, without using any electronic enhancement.

\section{Results}

The initial HLA-specific PCR assay was developed to investigate maternal microchimerism in a 49-year-old woman with scleroderma, and targeted the HLA class I antigen HLA-B44. The patient's class II haplotypes were $D R B 1 * 1104-D R B 3 * 02-D Q A 1 * 0501-D Q B 1 * 0301$ and $D R B 1 * 1501-D R B 5 * 0101-D Q A 1 * 0102-D Q B 1 * 0602$, and the mother was homozygous for the former haplotype; 
thus, no class II allele could be targeted to test for maternal DNA. However, at the HLA-B locus the mother had B44 and B8, whereas the patient had B35 and B8. The mother's and patient's HLA-B alleles were determined by sequencing to be $B * 4403,{ }^{*} 0801$, and $B^{*} 3508,{ }^{*} 0801$, respectively. HLA-B44-specific primers were developed, and the difference in B alleles were exploited to test for maternal-specific DNA in the patient. As seen in Figure 1, the 190-bp HLA-B44-specific PCR product was readily detected in DNA extracted from PBMCs of the patient. Blood samples from 3 different draw dates spanning 2.5 years ( 2 of which are shown in Figure 1 ) were positive for the noninherited maternal DNA. The HLAspecific PCR product was eluted, purified, and subjected to sequencing. The sequence obtained (Table 1 ) was identical to that from the mother (both from DNA extracted from PBMCs and from sequencing the product from the mother after the HLA-B44-specific assay). The sequence corresponds to amino acids 110-172 encoded by the third exon of $H L A-B * 4403$. No HLAB44-specific DNA was detected in another scleroderma patient for whom the mother had a nonshared HLA-B44 antigen. Two children represent alternative sources of microchimerism in this patient.

Additional HLA-specific assays were developed targeting HLA class II sequences of DRB5, DRB1*04, and $D R B 1 * 11$. The $D R B 5$ locus is absent on all HLA haplotypes, except for those that have $D R B 1$ alleles that encode for $D R 2$ molecules. Primers were developed that were specific for $D R B 5^{*} 01$, which is present on haplotypes that encode $D R B 1 * 15$, and were employed to test all scleroderma patients and healthy subjects for whom the mother had $D R B 5 * 01$ that was not shared by the subject. $D R 2$ molecules are encoded by $D R B 1 * 15$ and $D R B 1 * 16$ alleles; none of our families had $D R B 1 * 16$ alleles, so no other DRB5-encoded molecules were present in the families studied. $D R B 5 * 01$-specific DNA was detected in 2 of 3 SSC patients. $D R B 5^{*} 01$-specific DNA was also detected in 2 of 3 healthy normal subjects. The scleroderma patient shown in Figure 2 had positive results in all aliquots tested and in an additional assay (each assay is done in duplicate); the control subject had positive results in 1 aliquot but not in another aliquot from a different draw date. Sequencing of the 210-bp product revealed identity of the subjects and the mothers' alleles (Table 2). The sequence corresponds to amino acids 7-76 of $D R B 5 * 01$. In the scleroderma patient shown in Figure 2, $D R B 5 * 01$-specific DNA could also have originated from a child, because this patient had 3 sons, all of whom inherited $D R B 5 * 01$ from their father. However, the patient had no other pregnancies, and multiple tests for male DNA using both a quantitative assay (6) and a very sensitive nested PCR to test for male DNA (17) consistently revealed no male DNA in peripheral blood samples from this patient. In this scleroderma family, it is interesting that the haplotype the patient did not inherit from her mother was identical to the paternally inherited haplotype of her sons for $D R B 1, D R B 5, D Q A 1$, DQB1, and HLA-B.
$D R B 1 * 04$-specific and $D R B 1 * 11$-specific assays identified maternal microchimerism in 10 of 18 and 2 of 5 subjects, respectively. Two of 3 scleroderma patients had maternal-specific $D R B 1 * 04$ detected, as did 8 of 15 controls. In the normal subjects, the only potential source of $D R B 1 * 04$-specific DNA was from the mother. In 1 scleroderma patient, an alternative potential source was the patient's daughter, who inherited $D R B 1^{*} 04$ from her father. However, the child's $D R B 1 * 04$ allele was $D R B 1 * 0404$, whereas that of the patient's mother was $D R B 1 * 0401$. Additional testing was conducted in which a $D R B 1 * 04$-specific primer was used in conjunction with 1 of 2 different primers that target a difference between $D R B 1 * 0404$ and $D R B 1 * 0401$, based on a dimorphism at position 258 . The results clearly indicated that the chimeric DNA was $D R B 1 * 0401$ (maternal); no DRB1*0404 DNA was detected (data not shown). One scleroderma patient and 1 of 4 controls tested for $D R B 1 * 11$ had maternal microchimerism.

Overall maternal-specific DNA was detected in 17 of 31 (55\%) study subjects, without a significant difference in scleroderma patients and controls. The age range of scleroderma patients with persistent maternal microchimerism was 11-49 years (mean age: 34 years). The age range of all normal subjects with persistent maternal microchimerism was 9-46 years (mean age: 25 years).
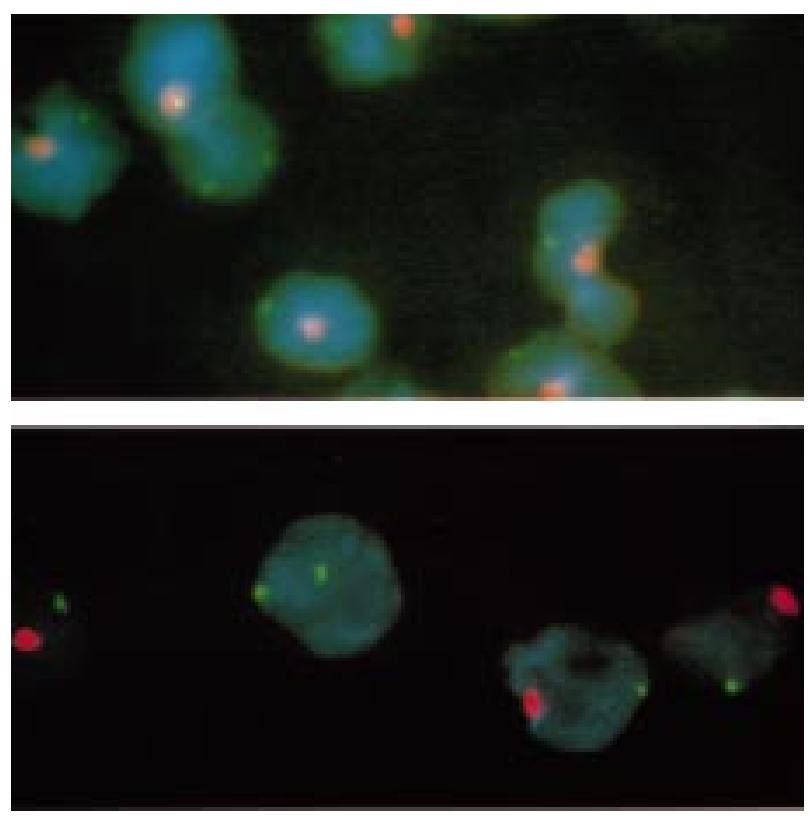

Figure 3

In situ hybridization with double labeling for $X$ and $Y$ chromosome-specific sequences of PBMCs from male patients with scleroderma (above) and scleroderma/myositis overlap (below). A cell with $2 \mathrm{X}$ chromosomes (green) is seen among male cells that contain both a Y chromosome (red) and an X chromosome (green) in each of the patients. (In the top panel, the $X$ and $Y$ signals are superimposed in the cell adjacent to the cell with $2 X$ signals.) The only known sources of female cells in the patients were their mothers. Neither patient had a history of blood transfusion or was a twin. $\times 100$. 
PBMC cytospin preparations from 5 male subjects were subjected to in situ hybridization with double labeling for $\mathrm{X}$ and $\mathrm{Y}$ chromosome-specific sequences. Male subjects included 3 scleroderma patients (1 with scleroderma/myositis overlap) and 2 normal subjects. Two female cells were seen in 1 of the scleroderma patients among 69,745 cells counted, and 1 female cell was seen among 88,567 cells counted in the patient with scleroderma/myositis overlap (Figure 3 ). No female cells were seen in a normal male among 32,622 cells counted, in a second normal male among 32,622 cells counted, or in the other male scleroderma patient among 40,687 cells counted.

\section{Discussion}

The frequency with which maternal cells pass into the fetal circulation was initially thought to be low, estimated at $1 \%$ in umbilical cord blood samples (18). Subsequently, Hall et al. (2) found maternal cells in $22 \%$ of cord blood samples. Other studies using PCR-based techniques increased the estimate to more than $40 \%$ (1). Maternal cells in cord blood could reflect events related to delivery. However, 2 recent studies have also found evidence of maternal cells in the fetal circulation in elective terminations in early and late gestation (19, 20). Maternal cells are known to engraft and persist in the circulation of infants with severe combined immunodeficiency $(4,21-26)$. However, to our knowledge, no previous study has specifically examined longterm persistence of maternal cells in immunologically competent offspring. Supporting the possibility, in an earlier study that used cytogenetic analysis, male infants who received in utero transfusion from their mothers were found to harbor up to $4 \%$ of lymphocytes, with $2 \mathrm{X}$ chromosomes at 4-5 years of age (27). In a murine model, maternal cells were found routinely in the bone marrow of immunocompetent fetuses (28).

We asked whether maternal microchimerism persists into adult life in patients with autoimmune disease and in normal individuals. To address this question, it was first necessary to develop sensitive techniques by which very small amounts of maternal DNA can be detected. For this purpose, HLA-specific PCR assays were designed and implemented to test study subjects for nonshared maternal-specific DNA. We found persistent maternal microchimerism in peripheral blood samples from scleroderma patients 11-49 years old. We also found persistent maternal microchimerism in normal subjects 9-46 years old. Products of HLA-specific PCR assays were subjected to sequencing, confirming their identity with maternal-specific HLA alleles.

Although scleroderma patients did not differ significantly from controls in the frequency with which maternal microchimerism could be detected, the finding that maternal microchimerism can persist through childhood and into adult life lends credence to the possibility that maternal microchimerism could play a role in some autoimmune diseases. In previous studies that investigated fetal microchimerism, we employed a quantitative assay for male DNA in women with sons and found a significant difference in quantitative levels of male DNA in scleroderma patients compared with healthy women (6). Scleroderma patients could also have quantitative differences of maternal microchimerism from normal subjects that would not be reflected in the current studies, because the HLAspecific assay is not quantitative. That maternal microchimerism could play a role in some systemic autoimmune diseases is supported by experimental models; for example, the introduction of parental cells into F1 progeny is a well-recognized model of systemic lupus erythematosus (29-32). Homing properties of chimeric cells could be important, as recently suggested by Aractangi et al. (33), who found fetal DNA in skin lesions of women with polymorphic eruptions of pregnancy, a skin disorder of pregnancy. Evidence for fetal cells in the skin of scleroderma patients has been described (34). Migration and localization may be key factors that determine the outcome of chimeric cells, as has recently been discussed (35). Maternal microchimerism could also be implicated in some neonatal and pediatric diseases.

The mechanisms by which a host maintains very low levels of nonhost cells over an extended period of time are unknown. In the current study, all mothers of subjects were HLA incompatible at 1 or more HLA loci and with few exceptions were HLA incompatible for multiple HLA class I alleles and HLA class II alleles. Tolerance to noninherited maternal HLA antigens has been described in highly sensitized patients awaiting kidney transplantation (36). A host could remain tolerant to noninherited maternal HLA alleles either because of in utero exposure or because of persistent microchimerism; long-lasting tolerance to fetal paternally inherited HLA alleles is also plausible. However, the finding that both maternal cells and fetal cells persist raises questions as to how differing microchimeric cell populations interact in the host and whether there are consequences to the host when particular HLA relationships occur between different chimeric cell populations. For example, in the current studies the noninherited maternal haplotype of 1 scleroderma patient was identical to the paternally inherited haplotype of her 3 sons for all class II alleles (also for HLA-B, but not for HLA-A). Immunological consequences of long-term maternal microchimerism could also differ from those of fetal microchimerism because, in the former, a fetus would be exposed during a time when the immune system is still developing. Factors that could influence potential immunological consequences of exposure to maternal cells include the timing of exposure, quantitative aspects of exposure, and medical conditions of pregnancy, i.e., preeclampsia (37).

In addition to persistent fetal microchimerism from pregnancy, another potential source of micro-chimerism is from a twin (8). One of the scleroderma patients who was tested for maternal microchimerism (and was negative) had an unaffected twin; however, the twin was HLA- 
identical and was not investigated further in these studies. Engraftment and persistence of donor cells after blood transfusion is another source of microchimerism, as was recently reported by Lee et al. (7). All subjects who previously received a blood transfusion were excluded from the current studies. Chimerism is also well described after organ transplantation, where it has been proposed as a mechanism for organ acceptance (38).

In summary, results of the current studies indicate that maternal cells can persist in immunocompetent progeny well into adult life and in the face of HLA incompatibility. How such low levels of nonhost cells are maintained in patients and in normal individuals is currently not known. The mechanisms of regulation and biological significance of long-term persistent microchimerism of maternal origin merit further investigation.

\section{Acknowledgments}

This work was supported by National Institutes of Health grants AI-38583, AI-14721, and AR-39282. The authors thank Tanya Hashizumi, Tracy Tylee, and Eileen Bryant for assistance with studies involving in situ hybridization; Effie Petersdorf for design of a primer and assistance with class I sequencing; and Anne Stevens, Bruce Gilliland, Jan Hillson, Julie Carkin Dale Reisner, Carol Wallace, and Jennifer Davis for referring patients to the study. Identification of male scleroderma patients by the Scleroderma Registry was greatly appreciated. The authors thank Jeff Hall for sharing experience and expertise in detection of maternal microchimerism, and Diana W. Bianchi for helpful comments.

1. Lo, Y.M.D., et al. 1996. Two-way cell traffic between mother and fetus: biologic and clinical implications. Blood. 88:4390-4395.

2. Hall, J.M., et al. 1995. Detection of maternal cells in human umbilical cord blood using fluorescence in situ hybridization. Blood. 86:2829-2832.

3. Bianchi, D.W., Zickwolf, G.K., Weil, G.J., Sylvester, S., and DeMaria, M.A. 1996. Male fetal progenitor cells persist in maternal blood for as long as 27 years postpartum. Proc. Natl. Acad. Sci. USA. 93:705-708.

4. Pollack, M.S., Kirpatrick, D., Kapoor, D., Supor, V., and O’Reilly, R.J. 1982. Identification by HLA typing of intrauterine derived maternal $T$ cells in four patients with severe combined immunodeficiency. N. Engl. J. Med. 307:662-666.

5. Nelson, J.L. 1996. Viewpoint. Maternal-fetal immunology and autoimmune disease. Is some autoimmune disease auto-alloimmune or alloautoimmune? Arthritis Rheum. 39:191-194.

6. Nelson, J.L., et al. 1998. Microchimerism and HLA-compatible relationships of pregnancy in scleroderma. Lancet. 351:559-562.

7. Lee, T.-H., Paglieroni, T., Ohto, H., Holland, P.V., and Busch, M.P. 1999. Survival of donor leukocyte subpopulations in immunocompetent transfusion recipients: frequent long-term microchimerism in severe trauma patients. Blood. 93:3127-3139.

8. DeMoor, G., Noens, L., and De Bic, S. 1988. A new case of human chimerism detected after pregnancy: 46, XY karyotype in the lymphocytes of a woman. Acta. Clin. Belg. 43:86-93.

9. Nelson, J.L., et al. 1993. Maternal-fetal disparity in HLA class II alloantigens and the pregnancy-induced amelioration of rheumatoid arthritis. N. Engl. J. Med. 329:466-471.

10. Mickelson, E., Smith, A., McKinney, S., Anderson, G., and Hansen, J.A. 1993. A comparative study of HLA-DRB1 typing by standard serology and hybridization of non-radioactive sequence-specific oligonucleotide probes to PCR-amplified DNA. Tissue Antigens. 41:86-93.

11. Smith, A.G., et al. 1996. Six new DR52-associated DRB1 alleles, three of DR8, two of DR11, and one of DR6, reflect a variety of mechanisms which generate polymorphism in the MHC. Tissue Antigens. 48:118-126.

12. Bodmer, J.G., et al. 1999. Nomenclature for factors of the HLA system 1998. Tissue Antigens. 53:407-446.

13. Petersdorf, E.W., and Hansen, J.A. 1995. A comprehensive approach for typing the alleles of the HLA-B locus by automated sequencing. Tissue Antigens. 46:73-85.

14. Schulman, H.M., et al. 1999. The biologic significance of rare peripheral blasts after hematopoietic cell transplant is predicted by multidimensional flow chemistry. Am. J. Clin. Pathol. In press.

15. Wayne, J.S., and Willard, H.F. 1985. Chromosome-specific alpha satellite DNA: nucleotide sequence analysis of the 2.0 kilobasepair repeat from the human X chromosome. Nucleic Acids Res. 13:2731-2743.

16. Lau, Y.F., Huang, J.C., Dozy, A.M., and Kan, Y.W. 1984. A rapid screening test for antenatal sex determination. Lancet. 1:14-16.

17. Evans, P.C., et al. 1999. Long-term fetal microchimerism in peripheral blood mononuclear cell subsets in healthy women and women with scleroderma. Blood. 93:2033-2037.

18. Socie, G., et al. 1994. Search for maternal cells in human umbilical cord blood by polymerase chain reaction amplification of two minisatellite sequences. Blood. 83:340-344.

19. Petit, T., et al. 1997. Detection of maternal cells in human fetal blood during the third trimester of pregnancy using allele-specific PCR amplification. Br. J. Haematol. 98:767-771.

20. Lo, Y.M.D., Hjelm, N.M., and Thilaganathan, B. 1998. Transfer of nucleated maternal cells into fetal circulation during the second trimester of pregnancy. Br. J. Haematol. 100:605-618.

21. Knobloch, C., Goldmann, S., and Friedrich, W. 1991. Limited T cell receptor diversity of transplacentally acquired maternal $\mathrm{T}$ cells in severe combined immunodeficiency. J. Immunol. 146:4157-4164.

22. Vaidya, S., et al. 1991. Suppression of graft-versus-host reaction in severe combined immunodeficiency with maternal-fetal T cell engraftment. Am. J. Pediatr. Hematol. Oncol. 13:172-175.

23. Wirt, D., et al. 1989. Novel T-lymphocyte population in combined immunodeficiency with features of graft-versus-host disease. $N$. Engl. J. Med. 321:370-374

24. Bastian, J.F., Williams, R.A., Ornelas, W., Tani, P., and Thompson, L.F. 1984. Maternal isoimmunization resulting in combined immunodeficiency and fatal graft-versus-host disease in an infant. Lancet. 1:1435-1437.

25. Pollack, M.S., et al. 1980. DR-positive maternal engrafted T cells in a severe combined immunodeficiency patient without graft-versus-host disease. Transplantation. 30:331-334.

26. Hansen, J.A., Good, R.A., and DuPont, B. 1977. HLA-D compatibility between parents and child: increased occurrence in severe combined immunodeficiency and other hematopoietic diseases. Transplantation. 23:366-374.

27. Hutchinson, D.L., Turner, J.H., and Schlesinger, E.R. 1971. Persistence of donor cells in neonates after fetal and exchange transfusion. Am.J. Obstet. Gynecol. 109:281-284.

28. Piotrowski, P., and Croy, B.A. 1996. Maternal cells are widely distributed in murine fetuses in utero. Biol. Reprod. 54:1103-1110.

29. Gleichman, E., Van Elven, H., and Van der Veen, J.P. 1982. A systemic lupus erythematosus (SLE)-like disease in mice induced by abnormal TB cell cooperation. Eur. J. Immunol. 12:152-155.

30. Portanova, J.P., Claman, H.N., and Kotzin, B.L. 1985. Autoimmunization in murine graft-vs-host disease. I. Selective production of antibodies to histones and DNA. J. Immunol. 135:3580-3586.

31. Portanova, J.P., Ebling, F.M., Hammond, W.S., Hahn, B.H., and Kotzin, B.L. 1988. Allogeneic MHC antigen requirements for lupus-like autoantibody production and nephritis in murine graft-vs-host disease. $J$. Immunol. 141:3370-3376.

32. Nelson, J.L. 1998. Microchimerism and autoimmune disease. N. Engl. J. Med. 338:1224-1225.

33. Aractingi, S., et al,. 1998. Fetal DNA in skin of polymorphic eruptions of pregnancy. Lancet. 352:1898-1901.

34. Artlett, C.M., Smith, B.J., and Jimenez, S. 1998. Identification of fetal DNA and cells in skin lesions from women with systemic sclerosis $N$. Engl.J. Med. 338:1186-1191.

35. Starzl, T.E., and Zinkernagel, R.M. 1998. Antigen localization and migration in immunity and tolerance. N. Engl. J. Med. 339:1905-1913.

36. Claas, F., Gijbels, Y., van der velden-de Munck, J., and van Rood, J. 1988. Induction of B cell unresponsiveness to noninherited maternal HLA antigens during fetal life. Science. 241:1815-1817.

37. Holzgreve, W., et al. 1998. Disturbed feto-maternal cell traffic in preeclampsia. Obstet. Gynecol. 91:669-672.

38. Starzl, T.E., et al. 1992. Systematic chimerism in human female recipients of male livers. Lancet. 340:876-877. 